\title{
Natural History and Conservative Management of Cubital Tunnel Syndrome
}

${ }^{a}$ Department of Orthopaedic Surgery, University of California, Davis School of Medicine, 4860 Y Street, Suite 3800, Sacramento, CA 95817, USA

${ }^{\mathrm{b}}$ Department of Orthopedics, University of California, Davis Medical Center, 2580 Stockton Boulevard, Sacramento, CA 95817, USA

Cubital tunnel syndrome is the second-most common nerve compression syndrome, second to carpal tunnel syndrome. It is, however, the most common site for ulnar nerve compression $[1,2]$. Accuracy in diagnosis is key in identifying the cubital tunnel as the site of compression, and, depending on the severity of the symptoms, nonoperative and operative treatment options have been proposed. In this article, we discuss the course of ulnar neuropathy caused by compression at the cubital tunnel and the conservative management of this syndrome.

\section{The natural course of cubital tunnel syndrome}

Buzzard [3], in 1922, described chronic neuritis of the elbow and attributed its causes to "excessive use of the hand and arm in flexed positions," ulnar nerve subluxation, and "some form of toxic agent." The term cubital tunnel was first proposed by Feindal and Stratford [4] in 1958. They emphasized that anatomic peculiarities that predispose the ulnar nerve to compression are present in this region of the elbow and noted a similarity between ulnar nerve compression at the elbow and median nerve compression in the carpal tunnel $[4,5]$. They observed the ulnar nerve being compressed in a fibro-osseous space defined by a ligament, which extends from the medial epicondyle to the olecranon. Its aponeuroticlike fibers adjoin

\footnotetext{
* Corresponding author.

E-mail address: rmszabo@ucdavis.edu (R.M. Szabo).
}

with the two heads of the flexor carpi ulnaris muscle $[4,6,7]$.

The elbow is a dynamic joint. Its arc of motion typically ranges from full extension to 150 degrees of flexion, with the functional range of motion being from 30 to 130 degrees. Throughout the day, the elbow flexes and extends to place the hand in positions of function. With motion, associated changes occur to the shape and space within the cubital tunnel.

The tunnel is most patent with the elbow in extension. With each degree of flexion, the tunnel changes its shape. Patel and colleagues [8], by using magnetic resonance imaging (MRI), showed that the tunnel is circular in shape and most spacious in extension. With flexion, the tunnel adopts a wider and flatter configuration. Beginning as a rounded tunnel, the tunnel becomes triangular or, as some describe, ellipsoid in flexion with a measurable height decrease of $2.5 \mathrm{~mm}$. Vanderpool and colleagues [9] showed that with the elbow in flexion, the aponeurosis stretches $5 \mathrm{~mm}$ for every 45 degrees of flexion. With the stretching of the aponeurosis and the innate tightness of the arcuate ligament, the tunnel has been shown to flatten and narrow by $55 \%$ with elbow flexion [10]. This decreases the space surrounding the nerve, making the nerve susceptible to compression $[4,9,11]$.

With the change in shape, an associated change in pressure occurs within the tunnel. Werner and colleagues [12] studied the cubital tunnel pressure measurements with the elbow in extension and flexion. The average pressure measured in extension was $9 \mathrm{~mm} \mathrm{Hg}$, and with flexion, the pressure increased to approximately $63 \mathrm{~mm} \mathrm{Hg}$. 
Q5] Gelberman [13] studied the extraneural and intraneural pressures and showed that with flexion, the extraneural pressure increased dramatically. Using cadaveric specimens, Gelberman showed that extraneural pressure measurements increased from 7 to $28 \mathrm{~mm} \mathrm{Hg}$ with flexion and intraneural pressure measurements increased from 8 to $41 \mathrm{~mm}$ $\mathrm{Hg}$ with flexion. Macnicol [14] also showed a rise in extraneural pressure with elbow flexion, recording pressure measurements as high as $200 \mathrm{~mm} \mathrm{Hg}$.

Q6 They also showed a decrease in pressure by $50 \%$ with release of the flexor aponeurosis. Iba and colleagues [7] studied cubital tunnel pressure measurements in patients and found that the highest extraneural pressure measurements were $1 \mathrm{~cm}$ distal from the proximal edge of the arcuate ligament. They also found that those with severe neuropathy had the highest pressure measurements with flexion.

As the cubital tunnel changes with elbow flexion, so does the ulnar nerve. With elbow flexion, the excursion of the nerve proximal to the medial epicondyle has been recorded to be as long as $10 \mathrm{~mm}$. Apfelberg and Larson [10] showed that the nerve elongates approximately $4.7 \mathrm{~mm}$ with the elbow in flexion. Distal to the medial epicondyle, the nerve stretches approximately 3 to $6 \mathrm{~mm}$ with flexion. Other studies have shown the nerve elongating even up to $8 \mathrm{~mm}$ [15]. This increases when the shoulder is held in abduction and the wrist is held in extension.

It is already clear that the environment surrounding the ulnar nerve at the elbow is a dynamic one. The natural course of the nerve is to experience some traction and some excursion with the elbow in motion. It is thought that with repetitive motion, the nerve becomes inflamed. With the inflammation comes edema and swelling in the nerve, which then affects its ability to glide. This has been well described both histologically and with imaging studies $[1,11,16]$. Studies have shown that blood flow and axonal transport are affected by compression [17-19]. With low external compression, extraneural and intraneural blood flow is impaired, leading to an increase in pressure and change in nerve conduction. With higher pressures, thickening in the nervous tissue and severe impairment in nerve conduction occur. Clark and colleagues [20] showed a decrease in neural blood flow with elongation. Using sciatic nerves in rats, they measured a $50 \%$ decrease in blood flow with $8 \%$ stretch of the nerve and an $80 \%$ reduction in nerve blood flow with $15 \%$ of elongation. Wall and colleagues [21] studied the effects of nerve conduction with stretch using tibial nerves. At $6 \%$ stretch, the nerve conduction decreased by $70 \%$; with $12 \%$ strain, conduction was completely blocked at 1 hour. Once decompressed, the recovery of nerve function was noted to be related to the severity and duration of the compression [2,20-23].

\section{Causes}

Cubital tunnel syndrome might be caused by constricting fascial bands, soft-tissue structures (hypertrophied synovium, tumor, ganglion, anconeus epitrochlearis muscle), bony abnormalities (cubitus valgus, bone spurs), or subluxation of the ulnar nerve over the medial epicondyle with elbow flexion. Although work-related activities involving repetitive elbow flexion and extension might aggravate cubital tunnel syndrome, no scientific data have supported work as a causal risk factor [24]. Many common themes are seen, however, in patients presenting with cubital tunnel syndrome. Environmental factors, such as specific occupations, have been associated with the diagnosis, and jobs that require repetitive motions involving elbow flexion have been implicated. Repetitive flexion can make one prone to developing traction neuritis because of the constant stretching of the ulnar nerve [25]. Baseball pitchers and tennis players often feel pain at the elbow and experience numbness in the ring and small fingers caused by the stress placed on the elbow. The wind-up while throwing a baseball or while serving a tennis ball stretches the ulnar nerve by placing a valgus force at the elbow while the shoulder is abducted. This position places the nerve under maximum compression and traction [26].

Similarly, people who partake in occupations such as carpentry, painting, and music typically are more prone to developing ulnar nerve symptoms, most commonly because of prolonged elbow flexion. Charness [27] reported that with 117 musicians, cubital tunnel syndrome was the most commonly diagnosed nerve entrapment syndrome. Another well-studied group involves wheelchair athletes, who are known to be prone to upper extremity injuries caused by repetitive impact and overuse. The prevalence of nerve entrapment in this group was $23 \%$. The majority of the neuropathies were from median nerve compression at the carpal tunnel. Thirty-nine percent of the nerve entrapment syndromes, however, involved the ulnar nerve, with a large subset of 
those patients having cubital tunnel syndrome. The strong forceful contractions of the flexor carpi ulnaris and the repetitive elbow flexion have been thought to cause compression of the ulnar nerve at the elbow [28].

Mechanical compression of the nerve at the elbow also is common because very little soft tissue surrounds the nerve. Postoperative ulnar nerve compression caused by inadequate padding at the elbow has been reported [29]. The superficial course of the ulnar nerve predisposes it to injury during patient positioning in the operating room. In the supine position, with the arm tucked to the side, direct compression can occur and will be accentuated if the arm slips slightly over the edge of the table. Wheelchair users who constantly place their elbows on the armrests often have symptoms of ulnar neuropathy [28]. Other sources include soft-tissue and bony abnormalities, such as cubitus valgus, ganglions, and space-occupying lesions in the cubital tunnel.

\section{Clinical presentation}

The most frequent way of diagnosing cubital tunnel syndrome is by obtaining a history and performing a physical examination. Patients commonly present with complaints of numbness and tingling in the small and ulnar half of the ring fingers, often accompanied by weakness of grip, particularly during activities for which torque is applied to a tool. Sensory involvement on the ulnar dorsal aspect of the hand also suggests cubital tunnel syndrome, as the dorsal cutaneous branch of the ulnar nerve originates proximal to the canal of Guyon. On rare occasions, patients present with wasting of the intrinsic musculature in the hand. Depending on the severity, the paresthesias might be intermittent or constant. The Semmes Weinstein monofilament test and vibration testing are helpful in detecting sensory impairment during the earlier stages of nerve compression. For more severe cases, static and moving two-point discrimination can be used. Weakness might also be present, although at times subtle. Comparison with the contralateral asymptomatic side can identify motor weakness with the intrinsic musculature. Patients also might complain of pain at the elbow and hypersensitivity with palpation of the ulnar nerve as it travels around the elbow [20-31]. In most cases, the forearm muscles are spared because their innervation might arise proximal to the cubital tunnel.
Weakness of the deep flexors to the ring and little fingers and weakness of the flexor carpi ulnaris, however, signal proximal ulnar nerve entrapment. Sunderland [32] studied the topography of the ulnar nerve at the level of the elbow and noted that the fascicles innervating the flexor carpi ulnaris and flexor digitorum profundus to the fourth and fifth digits are more central whereas the sensory fascicles and hand muscles are distributed more peripherally. The fibers are susceptible to changes in the environment, such as compression, frequent traction with elbow flexion, and direct trauma.

Provocative tests also are used to help identify ulnar neuropathy at the elbow. Tinel sign is positive when percussion of the ulnar nerve at the medial epicondyle reproduces paresthesias in the ring and small fingers. However, nearly $24 \%$ of asymptomatic people have this finding [33]. The elbow flexion test also has been used to corroborate the diagnosis of cubital tunnel syndrome. As described by Buehler [34], results of an elbow flexion test are positive when ulnar nerve symptoms are reproducible with the elbow flexed, the forearm supinated, and the wrist in extension for 3 minutes. Novak and colleagues [35] studied four different provocative tests, including Tinel sign, elbow flexion test, pressure provocation, and combined flexion with pressure provocation, and found that the combined test was the most sensitive and specific in diagnosing cubital tunnel syndrome. Only 2 of the 66 control participants experienced ulnar nerve symptoms with the combined test, whereas 43 of the 60 affected participants had positive results of the tests. Despite studies supporting the use of provocative tests, it is well known that the tests can also render positive results in asymptomatic people. The frequency of false positives has been reported with the use of the elbow flexion test and Tinel sign. With the wrist and shoulder in neutral position, Rayan and colleagues [33] showed that $10 \%$ of their asymptomatic patients had positive results of the flexion tests. The number of false positives increased when the test was performed with the shoulder abducted and the wrist extended. Therefore, care should be taken when interpreting the results of these tests, and an emphasis should be made on finding a positive correlation between the clinical examination and the history before making a diagnosis.

The use of electrodiagnostic testing can help locate, confirm, and quantify the severity of nerve compression. The ulnar nerve at the elbow has 
been studied, and guidelines have been formulated with the use of nerve conduction studies to aid in the diagnosis. Conduction velocities are measured across the elbow with the intrinsic musculature used for motor velocity and the small finger for sensory velocity. Parameters for accurate testing include flexing the elbow between 70 and 90 degrees when measuring conduction at the elbow [6]. The recommended length across the elbow is $100 \mathrm{~mm}$; however, studies have supported measuring 50 to $80 \mathrm{~mm}$ across the elbow to obtain the most accurate measurement of conduction [15]. The American Association of Electrodiagnostic Medicine criteria for a positive diagnosis of ulnar neuropathy include one of the following: absolute slowing of nerve conduction at the elbow, decreased conduction velocity of more than $10 \mathrm{~m} / \mathrm{s}$ across the elbow, decreased amplitude of more than $20 \%$, absence of sensory responses, or evidence of muscle atrophy [36]. Electromyography will reveal whether axonal degeneration has occurred. The first dorsal interosseous muscle is most commonly affected. The abductor pollicis brevis should be examined to exclude a C8-T1 nerve root or inferior brachial plexus lesion.

Radiographic examination of the elbow is useful in a small percentage of patients: those with arthritis, history of trauma, or abnormal elbow motion or carrying angle revealed by physical examination. Thoracic outlet syndrome most commonly involves the medial components of the brachial plexus and might be mistaken for cubital tunnel syndrome. An apical tumor of the lung can also compress or invade the inferior brachial plexus causing ulnar nerve symptoms. Chest radiography to rule out a Pancoast tumor should be obtained whenever a history of smoking, ulnar nerve symptoms, and shoulder pain is reported by the patient.

Ultrasonography also has been used to aid in the diagnosis of cubital tunnel syndrome. Studies have shown a difference in ulnar nerve size in patients diagnosed with cubital tunnel syndrome Q8 [37-39]. Wiesler and colleagues [38] showed that the affected patients had a statistically significant increase in the cross-sectional area of the nerve when compared with normal controls. The increase in size correlated with the idea that compression induced a cascade of events that included endoneurial edema and inflammation. The authors showed a positive correlation between nerve conduction studies and ultrasound measurements. Ultrasonography, therefore, might provide a noninvasive way to help diagnose cubital tunnel syndrome.
MRI has been studied as a modality with which to visualize changes to the ulnar nerve around the elbow [8]. Britz and colleagues [6] showed a strong correlation with positive MRI findings, such as an increase in signal around the nerve, and nerve compression. The authors found that MRI was more sensitive than electrodiagnostic studies in diagnosing ulnar nerve compression at the elbow.

\section{Staging}

In 1950, McGowen [22] introduced a staging system that solely reflected the motor aspect of the ulnar nerve. Grade I had undetectable motor weakness. Grade II showed some motor weakness, and Grade III was described as severe motor weakness. Sensory findings were later included into the staging classification. For mild cases, symptoms are intermittent and include occasional paresthesia. The patient might have complaints of weakness, but findings of the motor examination typically are normal. For moderate cases, paresthesias are intermittent, and clinically, a decrease in vibratory sensation might be present. A discrepancy in intrinsic strength when compared with the unaffected side might be present. Results of provocative tests are also positive. In more severe cases, patients might complain of constant numbness in the ulnar nerve distribution in the hand and might have abnormal results of the two-point discrimination test. The patients present with atrophy of the ulnar innervated intrinsics and have obvious weakness.

Dellon [2] and Gabel [40] developed more comprehensive staging classifications by using additional diagnostic criteria and creating a more precise tool for reporting research data. Dellon [41] used a numeric grading scale to categorize patients based on symptoms. A numeric score of 0 indicated normal results. Scores of 1 and 2 included intermittent paraesthesias and mild weakness observed during pinch and grip tests. Scores of 3 and 4 included vibratory changes and moderate objective weakness. A score of 5 indicated persistent paresthesias, and a score of 6 showed abnormal two-point discrimination. Scores 7 through 10 evidenced muscle atrophy. Based on the numeric grading, the authors showed that mildly affected patients achieved better outcomes with conservative therapy, whereas those with higher scores were more likely to need surgical treatment. This confirmed the findings of an earlier study in which 
Dellon [2] found that for $50 \%$ of patients with a minimal degree of nerve compression, excellent results were achieved by using nonoperative techniques.

\section{Conservative therapy}

Conservative therapy has been proposed and adopted for patients presenting with mild symptoms $[2,31,42,43]$. The goals of the treatment are to eliminate or decrease the frequency of symptoms and to prevent further progression of the disease. A detailed history is important and helps identify the activities that aggravate the symptoms. Patient education plays an important part in treating the mild symptoms. Activities that reproduce symptoms such as repetitive elbow flexion or direct pressure to the medial epicondyle should be avoided or limited, and elimination of these inciting activities has been shown to provide relief. The patient might be required to modify habits and the work environment $[43,44]$.

In conjunction with activity modification, splinting has played a successful role in the conservative management of cubital tunnel syndrome. An elbow pad can help prevent direct trauma to the nerve. Wearing a splint also can act as a reminder to the patient to avoid flexing the elbow. By limiting flexion to 45 to 70 degrees, Q11] Dimond [15] and Lister [45] reported an 86\% Q12] improvement of symptom severity in 73 patients who underwent splinting during an average 8.7 months. Studies have shown as high as $90 \%$ successful treatment achieved by using conservative modalities [15,45].

Beekman and colleagues [46] prospectively studied 74 patients who had cubital tunnel syndrome. Based on diagnostic testing, the authors divided the patients into two treatment groups. Forty-six patients were treated conservatively, and 28 were treated surgically. The division was decided based on the presenting symptoms, with the conservative group having more mild symptoms, limited to intermittent paresthesias and mild intrinsic weakness. The instructions for conservative treatment included avoiding leaning on the elbow, avoiding crossing the arms while sitting, and keeping the elbow extended as much as possible. After the 6 months of treatment, 35\% of the conservatively treated patients achieved improvement and $11 \%$ experienced complete remission. The authors noted that during the course of 6 months, those with only sensory symptoms did not progress with any worsening symptoms or any motor involvement, thus implying that treatment can halt the progression of the neuropathy.

Night splinting has been successfully used in patients presenting with cubital tunnel syndrome. Seror and colleagues [47] studied 22 patients with electrodiagnostically confirmed ulnar nerve palsy and treated them with night splints. The splints limited flexion from 15 to 60 degrees but allowed unrestricted pronation and supination. During the daytime, the patients were advised not to rest the elbow on hard surfaces or partake in prolonged elbow flexion. The splints were worn regularly for 6 months. After 11.3 months, fewer symptoms were present in every patient treated and five of the patients reported $80 \%$ to $90 \%$ subjective improvement in symptoms. Sixteen of the 17 patients additionally experienced electrodiagnostic improvement. The best responders for the treatment were those who underwent splinting less than 3 weeks after the onset of symptoms. The first symptom to resolve was nocturnal paresthesias. Those more severely affected also showed improvements with sensation and strength; however, the authors noted that the time to recovery was more prolonged compared with the mildly affected patients. Interestingly, three of the patients included in the study had undergone previous surgical decompressions that did not achieve resolution of symptoms. Nighttime splinting did improve symptoms in all three patients, and clinical improvement for the three ranged from $60 \%$ to $95 \%$. Two of the patients additionally had improvement shown by electromyography.

Dellon and colleagues [41] prospectively studied 121 patients treated nonoperatively for a minimum of 3 months up to 6 months. The nonoperative management included thermoplastic splints or towel wrapping at night, patient education, and work modification. Alterations included placing pillows under the elbow for computer users. Telephones were to be used with the contralateral arm. Crossing arms was avoided, and patients were taught to place their hands on their thighs with their forearms supinated. Of the patients with intermittent paresthesias, $42 \%$ became symptom free after conservative treatment. Thirty-four percent of patients with moderate symptoms, which included electromyographic findings but no clinical signs of intrinsic wasting or abnormal two-point discrimination, became symptom free after 6 months of treatment. Twenty percent of the more severely affected patients also reported 
being symptom free. The need for surgical treatment was $21 \%$ within 6 years for patients with mild symptoms, 33\% within 3 years for moderate symptoms, and $62 \%$ within 3 years for severe symptoms.

Many varieties of splints are available. Homemade, custom fitted, and pre-made commercially available splints have been used to prevent elbow flexion. Apfel and Sigafoos [48] studied five different types of splints, four of which are commercially available, to learn how effective they are in preventing flexion. The five splints consisted of the following: a large bath towel applied circumferentially around the elbow joint; Pil-O Splint elbow support adjustable with rigid plastic stay (IMAK Corp., San Diego, CA); Pil-O-Splint elbow support adjustable with rigid plastic stay removed (IMAK Corp.); the Hely \& Weber cubital tunnel splint (Hely \& Weber, Santa Paula, CA); and the AliMed Cubital Tunnel Syndrome Support (AliMed, Inc., Dedham, MA). Using cadaveric limbs, the authors studied the splints' ability to prevent elbow flexion against gravity and with an added weight. They found that the AliMed splint allowed for the most flexion against gravity, allowing the elbow to flex to 110 degrees. The Hely \& Weber splint allowed only 53 degrees of flexion. This splint also prevented full extension: on average, 17 degrees of extension. The remaining splints allowed for elbow flexion, although none exceeded 90 degrees. By preventing the elbow to bend beyond 90 degrees, the products minimized compression on the ulnar nerve.

A consensus exists that limiting elbow flexion is what makes splinting effective; however, the degree of flexion that is tolerable has not been established. Gelberman [43] showed that the lowest mean extraneural and intraneural pressures of the ulnar nerve occurred when the elbow was flexed to 40 to 50 degrees. The highest pressures were recorded with the elbow in maximal flexion, which was approximately 130 degrees. Interestingly, the elbow in full extension also recorded higher pressures at the cubital tunnel than when the elbow was flexed between 30 and 70 degrees [13]. Hong and colleagues [49] recommended limiting flexion to 35 degrees, whereas other studies $[1,50]$ reported using 45 degrees as the limit. Although it seems the studies used different parameters for splinting, an underlying consistency exists in that a slight amount of flexion is more beneficial in decreasing the pressure in the cubital tunnel and that patients find slight flexion more tolerable than full extension [43].
Little support is offered for local steroid injections into the cubital tunnel. Unlike with carpal tunnel syndrome, the response to cubital tunnel syndrome from steroid injections has not been as beneficial [37,44]. Hong and colleagues [48] studied 12 ulnar nerves, dividing the patients into two groups. Group A was treated with nocturnal and intermittent daytime splinting, limiting elbow flexion to 35 degrees. The splint was molded to 30 to 35 degrees of elbow flexion, forearm to 10 to 20 degrees of pronation, and wrist held in neutral. At the elbow, padding was added with added space medially. Compliance was monitored closely during the course of 6 months. Group B was treated with a similar splinting regimen and local steroid injection. The follow-up duration was 6 months, and the results showed that splinting alone was sufficient for treating mild symptoms. The addition of the steroid injection did not provide any further improvement in sensory or motor conduction. After 1 month of treatment, Group A participants reported symptomatic improvement and showed improvement in motor conduction, whereas Group B participants did not show any improvement in motor conduction at that time. At 6 months, however, a significant decrease in conduction time was shown in both groups. No change in sensory conduction occurred in either group at 1 or 6 months, most likely because recovery of the sensory type Ia fibers took longer than 6 months [49]. It is important to view all the findings in perspective with the natural history of untreated cubital tunnel syndrome with which approximately half of patients improve spontaneously [51].

\section{Summary}

Conservative therapy with splinting is an effective way to treat cubital tunnel syndrome. The variables of splinting include the type and durability of the splint and the patient's compliance. Factors such as comfort, practicality, and cosmesis play a large role in a patient's compliance with the treatment protocol $[45,48,50]$. Likewise, the rigidity of the splint, preventing flexion at angles beyond 90 degrees, is a very important factor in the success of the management of mild cubital tunnel syndrome. Although a consensus regarding duration of treatment, type of splinting, and degree of splinting is lacking, overall, a consistency in the support of the effectiveness of this modality in the spectrum of treatment options for cubital tunnel syndrome exists. 


\section{References}

[1] Bozentka DJ. Cubital tunnel syndrome pathophysiology. Clin Orthop Relat Res 1998;351:90-4.

[2] Dellon AL. Review of treatment results for ulnar nerve entrapment at the elbow. J Hand Surg [Am] 1989;14(4):688-700.

[3] Buzzard E. Some varieties of traumatic and toxic ulnar neuritis. Lancet 1922;1(Feb 18):317-9.

[4] Feindel W, Stratford J. The role of the cubital tunnel in tardy ulnar palsy. Can J Surg 1958;1(4):287-300.

[5] Feindel W, Stratford J. Cubital tunnel compression in tardy ulnar palsy. Can Med Assoc J 1958;78(5):351-3.

[6] Britz GW, Haynor DR, Kuntz C, et al. Ulnar nerve entrapment at the elbow: correlation of magnetic resonance imaging, clinical, electrodiagnostic, and intraoperative findings. Neurosurgery 1996;38(3): 458-65.

[7] Iba K, Wada T, Aoki M, et al. Intraoperative measurement of pressure adjacent to the ulnar nerve in patients with cubital tunnel syndrome. J Hand Surg [Am] 2006;31(4):553-8.

[8] Patel VV, Heidenreich FP Jr, Bindra RR, et al. Morphologic changes in the ulnar nerve at the elbow with flexion and extension: a magnetic resonance imaging study with 3-dimensional reconstruction. J Shoulder Elbow Surg 1998;7(4):368-74.

[9] Vanderpool DW, Chalmers J, Lamb DW, et al. Peripheral compression lesions of the ulnar nerve. J Bone Joint Surg Br 1968;50(4):792-803.

[10] Apfelberg DB, Larson SJ. Dynamic anatomy of the ulnar nerve at the elbow. Plast Reconstr Surg 1973; 51(1):79-81.

[11] Pechan J, Julis I. The pressure measurement in the ulnar nerve: a contribution to the pathophysiology of the cubital tunnel syndrome. J Biomech 1975; $8(1): 75-9$.

[12] Werner CO, Ohlin P, Elmqvist D. Pressures recorded in ulnar neuropathy. Acta Orthop Scand 1985;56(5):404-6.

[13] Gelberman RH, Yamaguchi K, Hollstien SB, et al. Changes in interstitial pressure and cross-sectional area of the cubital tunnel and of the ulnar nerve with flexion of the elbow: an experimental study in human cadavera. J Bone Joint Surg Am 1998; 80(4):492-501.

[14] Macnicol MF. Extraneural pressures affecting the ulnar nerve at the elbow. Hand 1982;14(1):5-11.

[15] Kern RZ. The electrodiagnosis of ulnar nerve entrapment at the elbow. Can J Neurol Sci 2003; 30(4):314-9.

[16] Green JR Jr, Rayan GM. The cubital tunnel: anatomic, histologic, and biomechanical study. J Shoulder Elbow Surg 1999;8(5):466-70.

[17] Aziz W, Firrell JC, Ogden L, et al. Blood flow in a chronic entrapment neuropathy model in the rabbit sciatic nerve. J Reconstr Microsurg 1999;15(1): 47-53.
[18] Lundborg G, Dahlin LB. The pathophysiology of nerve compression. Hand Clin 1992;8(2):215-27.

[19] Dahlin LB, Rydevik B, McLean WG, et al. Changes in fast axonal transport during experimental nerve compression at low pressures. Exp Neurol 1984; 84(1):29-36.

[20] Clark WL, Trumble TE, Swiontkowski MF, et al. Nerve tension and blood flow in a rat model of immediate and delayed repairs. J Hand Surg [Am] 1992;17(4):677-87.

[21] Wall EJ, Massie JB, Kwan MK, et al. Experimental stretch neuropathy: changes in nerve conduction under tension. J Bone Joint Surg Br 1992;74(1):126-9.

[22] McGowen A. The results of transposition of the ulnar nerve for traumatic ulnar neuritis. J Bone Joint Surg Br 1950;32(3):293-301.

[23] Posner MA. Compressive neuropathies of the ulnar nerve at the elbow and wrist. Instr Course Lect 2000; 49:305-17.

[24] Blair W. Cubital tunnel syndrome in the work environment. In: Gordon S, Blair S, Fine L, editors. Repetitive motion disorders of the upper extremity. Rosemont (IL): American Academy of Orthopaedic Surgery; 1995. p. 455-65.

[25] Terrono AL, Millender LH. Management of workrelated upper-extremity nerve entrapments. Orthop Clin North Am 1996;27(4):783-93.

[26] Aoki M, Takasaki H, Muraki T, et al. Strain on the ulnar nerve at the elbow and wrist during throwing motion. J Bone Joint Surg Am 2005;87(11): 2508-14.

[27] Charness M. Unique upper extremity disorders of musicians. In: Millender L, Louis D, Simmons B, editors. Occupational disorders of the upper extremity. New York: Churchill Livingstone; 1992. p. 227-52.

[28] Burnham RS, Steadward RD. Upper extremity peripheral nerve entrapments among wheelchair athletes: prevalence, location, and risk factors. Arch Phys Med Rehabil 1994;75(5):519-24.

[29] Miller RG, Camp PE. Postoperative ulnar neuropathy. JAMA 1979;242(15):1636-9.

[30] Cooper D. Nerve injury associated with patient positioning in the operating room. In: Gelberman R, editor. 1 st editon. Operative nerve repair and reconstruction. vol. 2. Philadelphia: JB Lippincott; 1991. p. 1231-42.

[31] Idler RS. General principles of patient evaluation and nonoperative management of cubital syndrome. Hand Clin 1996;12(2):397-403.

[32] Sunderland S. The intraneural topography of the radial, median and ulnar nerves. Brain 1945;68: 243-99.

[33] Rayan GM, Jensen C, Duke J. Elbow flexion test in the normal population. J Hand Surg [Am] 1992; 17(1):86-9.

[34] Buehler MJ, Thayer DT. The elbow flexion test. A clinical test for the cubital tunnel syndrome. Clin Orthop Relat Res 1988;233:213-6. 
[35] Novak CB, Lee GW, Mackinnon SE, et al. Provocative testing for cubital tunnel syndrome. J Hand Surg [Am] 1994;19(5):817-20.

[36] American Association of Electrodiagnostic Medicine, American Academy of Neurology, American Academy of Physical Medicine and Rehabilitation. Practice parameter for electrodiagnostic studies in ulnar neuropathy at the elbow: summary statement. Muscle Nerve 1999;22(3):408-11.

[37] McPherson SA, Meals RA. Cubital tunnel syndrome. Orthop Clin North Am 1992;23(1):111-23.

[38] Park GY, Kim JM, Lee SM. The ultrasonographic and electrodiagnostic findings of ulnar neuropathy at the elbow. Arch Phys Med Rehabil 2004;85(6): 1000-5.

[39] Wiesler ER, Chloros GD, Cartwright MS, et al. Ultrasound in the diagnosis of ulnar neuropathy at the cubital tunnel. J Hand Surg [Am] 2006;31(7): 1088-93.

[40] Gabel G, Amadio P. Reoperation for failed decompression of the ulnar nerve in the region of the elbow. J Bone Joint Surg Am 1990;72(2):213-9.

[41] Dellon AL, Hament W, Gittelshon A. Nonoperative management of cubital tunnel syndrome: an 8-year prospective study. Neurology 1993;43(9): 1673-7.

[42] Eisen A, Danon J. The mild cubital tunnel syndrome: its natural history and indications for surgical intervention. Neurology 1974;24(7):608-13.

[43] Blackmore S. Therapist's management of ulnar nerve neuropathy at the elbow. In: Mackin E, editor.
Rehabilitation of the hand and upper extremity. St Louis (MO): Mosby; 2002. p. 679-89.

[44] Lund AT, Amadio PC. Treatment of cubital tunnel syndrome: perspectives for the therapist. J Hand Ther 2006;19(2):170-8.

[45] Dimond M, Lister G. Cubital tunnel syndrome treated by long-term splintage. Proceedings of the 1985 American Society for Surgery of the Hand Annual Meeting. J Hand Surg [Am] 1985;10:430.

[46] Beekman R, Wokke JH, Schoemaker MC, et al. Ulnar neuropathy at the elbow: follow-up and prognostic factors determining outcome. Neurology 2004;63(9):1675-80.

[47] Seror P. Treatment of ulnar nerve palsy at the elbow with a night splint. J Bone Joint Surg Br 1993;75(2): 322-7.

[48] Apfel E, Sigafoos GT. Comparison of range-of-motion constraints provided by splints used in the treatment of cubital tunnel syndrome: a pilot study. J Hand Ther 2006;19(4):384-92.

[49] Hong CZ, Long HA, Kanakamedala RV, et al. Splinting and local steroid injection for the treatment of ulnar neuropathy at the elbow: clinical and electrophysiological evaluation. Arch Phys Med Rehabil 1996;77(6):573-7.

[50] Sailer SM. The role of splinting and rehabilitation in the treatment of carpal and cubital tunnel syndromes. Hand Clin 1996;12(2):223-41.

[51] Padua L, Aprile I, Caliandro P, et al. Natural history of ulnar entrapment at elbow. Clin Neurophysiol 2002;113(12):1980-4. 